\title{
Síndrome de Kounis: una posibilidad a tener en cuenta como causa de muerte súbita cardiaca
}

\section{Kounis syndrome: a possibility to be considered as a cause of sudden cardiac death}

\begin{abstract}
Resumen
El llamado síndrome miocárdico alérgico, angina alérgica o síndrome de Kounis, se define como la aparición de un síndrome coronario agudo por vasoespasmo secundario a la activación de mastocitos y células inflamatorias interrelacionadas que se produce en las reacciones de hipersensibilidad, anafilácticas y anafilactoides. Presentamos un caso de muerte súbita en un adulto joven compatible con un síndrome de Kounis, donde el estudio histopatológico demuestra patología asmática y una trombosis en la arteria coronaria descendente anterior sobre una placa erosionada con numerosos eosinófilos y mastocitos en el trombo, en la placa subyacente, en la pared muscular y en la adventicia. La entrevista familiar posterior confirmó el antecedente de atopia y una clínica previa al fallecimiento compatible con un episodio asmático. Como dicen muchos expertos, el Síndrome de Kounis no es una enfermedad rara, pero sí es una enfermedad raramente diagnosticada. Debemos tenerla presente y descartarla ante una muerte súbita en el contexto de una reacción alérgica o de antecedentes de atopia, alergia o asma alérgico.
\end{abstract}

Palabras clave: Anafilaxia. Angina alérgica. Muerte súbita. Síndrome de Kounis.

\section{Abstract}

Kounis syndrome has been defined as the concurrence of an acute coronary syndromes with allergic or hypersensitivity as well as with anaphylactic or anaphylactoid reactions. The main pathophysiological mechanism is the vasospasm of epicardial coronary arteries due to increased inflammatory mediators that are released during the underlaying allergic events. We present a case of sudden death in an atopic young adult with histopathological findings of asthma and thrombosis in left anterior descending coronary artery with presence of eosinophils and mast cell. Kounis syndrome is not a rare disease but is a rarely diagnosed condition which should always be kept in mind when dealing with a cardiac sudden death with clinical history of atopy or allergy or in a context of allergic reactions.

Key words: Anaphylaxis. Allergic angina. Sudden death. Kounis syndrome.

\section{Introducción}

El Síndrome de Kounis, también conocido como Síndrome Anginoso Alérgico, se define como un síndrome coronario agudo provocado por la liberación de mediadores inflamatorios en una reacción de hipersensibilidad inmediata anafiláctica o una reacción anafilactoide. Fue descrito en 1991 por Kounis y Zavras ${ }^{1}$. Se han descrito tres variantes del síndrome ${ }^{2}$ : el tipo I se presenta en pacientes con arterias coronarias normales sin factores predisponentes de enfermedad coronaria (las reacciones de hipersensibilidad producirían un espasmo coronario o un infarto por disfunción endotelial) y el tipo II, en pacientes con enfermedad ateromatosa, en los cuales, el espasmo coronario puede inducir erosión o rotura de la placa con la consiguiente trombosis coronaria. El tipo III, recientemente descrito ${ }^{3}$, se presenta en pacientes con trombosis sobre un stent coronario farmacoactivo en donde se demuestra la presencia de eosinófilos y mastocitos en el material trombótico aspirado.

Se presenta un caso de muerte súbita en el que el estudio histopatológico y la investigación posterior de las circunstancias de la muerte apoyan la posibilidad del Síndrome de Kounis como causa de la muerte.

\author{
P. Molina ${ }^{1}$ \\ C. Presentación ${ }^{2}$ \\ P. Beltrán ${ }^{2}$ \\ E. Zorio ${ }^{3}$
}

${ }^{1}$ Facultativo especialista en Anatomía Patológica. Instituto de Medicina Legal de Valencia. ${ }^{2}$ Médico Forense. Especialista en Medicina Legal y Forense.

Instituto de Medicina Legal de Valencia.

${ }^{3}$ Facultativo especialista en Cardiología.

Hospital Universitario y Politécnico La Fe de Valencia.

\section{Correspondencia: Pilar Molina Aguilar Servicio de Patología. Instituto de Medicina Legal Ciudad de la Justicia. Avenida del Saler, 14. 46013 Valencia E-mail:molina_pil@gva.es}

Fecha de recepción: 22.FEB.2012

Fecha de aceptación: 18.MAR.2012 


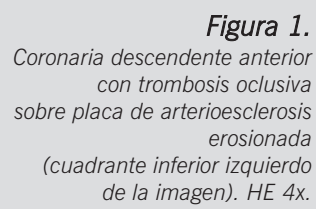

Figura 1.

Coronaria descendente anterior con trombosis oclusiva sobre placa de arterioesclerosis erosionada

(cuadrante inferior izquierdo de la imagen). HE $4 x$

Figura 2. Detalle de la celularidad inflamatoria con predominio de eosinófilos en el interior del trombo organizado. HE 40X.
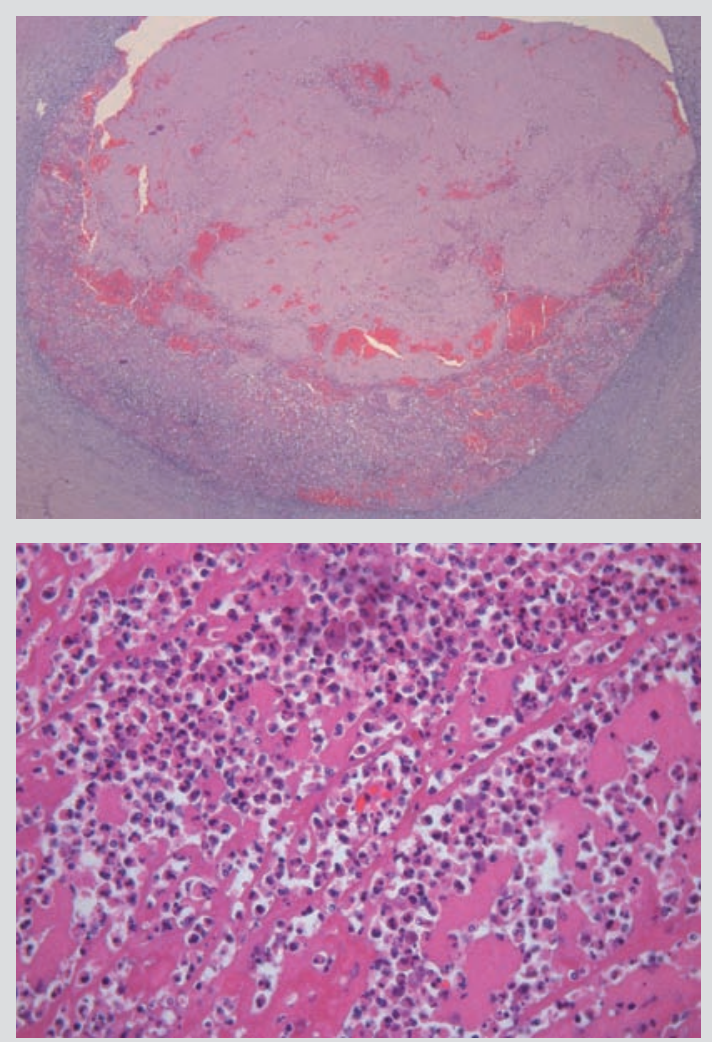

Figura 3.

Presencia de numerosos mastocitos en la placa de ateroma en un área cercana a la superficie erosionada. Se ha realizado una tinción con Azul de metileno para visualizar los gránulos metacromáticos en el citoplasma de los mastocitos parcialmente degranulados (flechas)

o liberados en la placa. 40x.

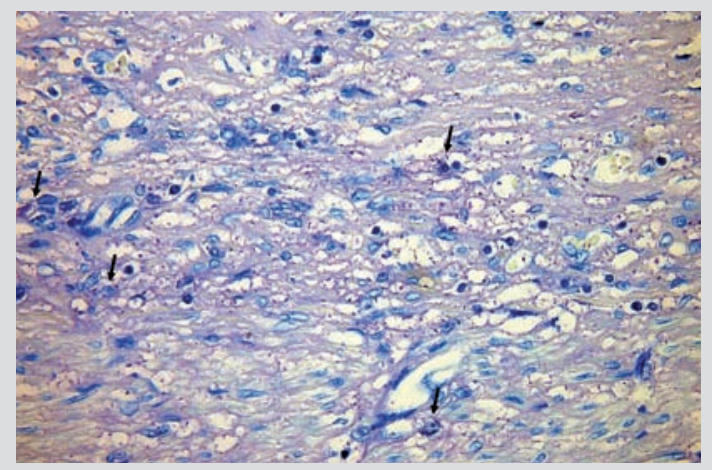

Figura 4. Corte histológico del pulmón con obstrucción bronquial por tapones de moco, severo infiltrado inflamatorio en lámina propia y en submucosa, engrosamiento de la membrana basal e hipertrofia muscular parietal. HE 4x

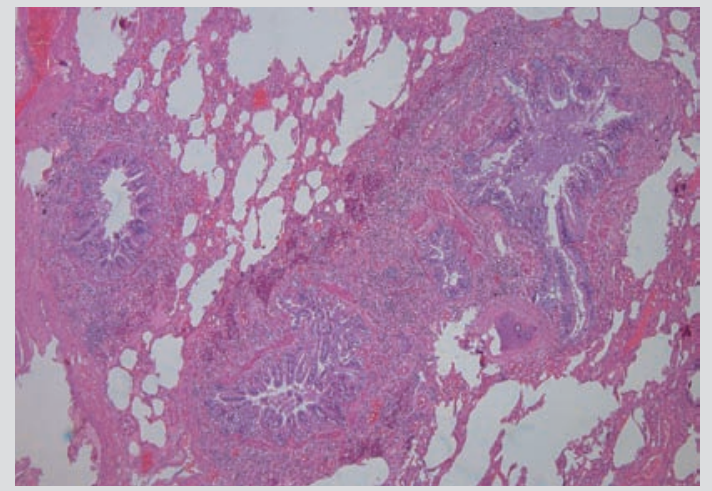

\section{Presentación del caso}

Autopsia judicial de un caso de muerte súbita en un hombre de 37 años durante su actividad cotidiana, con estudios complementarios (toxicológicos e histopatológicos) y entrevista familiar.

Estudio postmortem: hallazgos destacados del examen interno: corazón de 389 g de peso (valor medio según peso corporal: 345 g, rango 261-455 g). Grosor pared ventrículo izquierdo: $1,3 \mathrm{~cm}$, tabique interventricular: $1,3 \mathrm{~cm}$ y ventrículo derecho: $0,2 \mathrm{~cm}$. Agujero oval no permeable. Aparato valvular y miocardio sin lesiones. Dominancia derecha. Arterioesclerosis coronaria con estenosis aproximadamente del $50 \%$ del calibre de la arteria coronaria descendente anterior con trombosis de la misma. Esófago anillado (1 cm) en su tercio distal (Figs. 1-6).

El estudio histológico confirma el grado de arterioesclerosis y la trombosis coronaria oclusiva en la arteria descendente anterior con infiltración inflamatoria en la placa y adventicia, compuesta principalmente por eosinófilos y mastocitos. Se demuestra asimismo, patología pulmonar compatible con asma bronquial y esofagitis crónica con infiltrado eosinófilo, sin cumplir criterios de esofagitis eosinófila (menor a 24 por campo de gran aumento). El estudio toxicológico postmortem es negativo. La entrevista familiar confirma el antecedente de atopia y una sintomatología previa a la muerte compatible con una crisis asmática.

\section{Discusión}

Las reacciones de hipersensibilidad asociadas a un síndrome coronario no son raras, pero siguen estando poco documentadas en la literatura médica. El llamado síndrome miocárdico alérgico, angina alérgica o síndrome de Kounis, se define como la aparición de un síndrome coronario agudo secundario a la activación de mastocitos y células inflamatorias interrelacionadas que puede aparecer en el contexto de reacciones de hipersensibilidad anafilácticas y anafilactoides. En la práctica clínica, cada vez se describen más casos de síndrome de Kounis ante diferentes desencadenantes que incluyen: fárma$\cos ^{4,5,6}$ (antibióticos, analgésicos, AINES, corticoides, anestésicos intravenosos, medios de contraste, anticoagulantes, desinfectantes cutáneos, antineoplásicos, trombolíticos), angioedema, asma bronquial, urticaria, alergia alimentaria, anafilaxia inducida por el ejercicio físico, mastocitosis, implantación de stent farmacoactivos ${ }^{7}$, parches de nicotina ${ }^{8}$, marisco ${ }^{9}$, picaduras de insectos ${ }^{10,11}$, etc. 
Los mastocitos o células cebadas se localizan en el tejido conjuntivo de los diferentes órganos o sistemas y su activación y degranulación produce las reacciones alérgicas y anafilactoides. Los mediadores preformados, como serotonina, histamina y enzimas (triptasa, quimasa, $\beta$-hexosaminidasa), se almacenan en gránulos citoplasmáticos que son liberados por exocitosis (degranulación). Los mediadores de nueva síntesis incluyen citoquinas, quimioquinas, factores de crecimiento y productos del metabolismo del ácido araquidónico. Además, los mastocitos constituyen el único sistema renina-angiotensina extrarrenal constituyendo una fuente adicional de renina ${ }^{12,13}$.

Los mecanismos por los cuales las sustancias liberadas participan en la fisiopatología del síndrome coronario agudo en el síndrome de Kounis se describen a continuación. Los metabolitos del ácido araquidónico (leucotrienos y tromboxano) y la histamina pueden inducir espasmo coronario e infarto agudo de miocardio. La histamina además, produce activación y agregación plaquetaria, modula la respuesta inflamatoria de los neutrófilos, monocitos y esosinofilos, induce la producción de citoquinas por las células endoteliales, y a nivel experimental, se ha visto que induce engrosamiento de la íntima en ratones ${ }^{4,5}$. La triptasa y quimasa al activar las metaloproteinasas (colagenasa, gelatinasa y estromelisin) localizadas en la placa ateromatosa, juegan un papel importante en la erosión y ruptura de la misma ${ }^{14}$. La quimasa es un convertidor de la angiotensina I en angiotensina II, que actúa sobre las células musculares de la capa media de las coronarias agravando el espasmo coronario producido por la histamina y los leucotrienos ${ }^{15}$.

Algunos de los casos de angina inestable o infarto de miocardio en la población general son el resultado de una trombosis coronaria por la combinación de un espasmo coronario y la erosión o rotura de una placa ateromatosa. Se ha comprobado que en estos pacientes hay también un aumento en sangre y orina de los mediadores de las reacciones alérgicas agudas (histamina, triptasa, interleukina-6) ${ }^{16,17}$ y existen fuertes evidencias que sugieren que tanto el infiltrado inflamatorio en las placas como la activación de mastocitos preceden al episodio coronario agudo ${ }^{18}$, pudiendo indicar una vía común y una mayor incidencia de síndromes coronarios en individuos atópicos. Por otro lado, se ha demostrado, experimental y clínicamente, que el corazón puede ser el primer órgano diana de la anafilaxia, produciéndose una primera fase de fallo cardiaco agudo por la liberación intracardiaca de histamina y una segunda fase sistémica, donde la vasodilatación periférica, la disminución del retorno venoso, la pérdida de volumen debido a la aumento de la permeabilidad vascular y la consiguiente depresión del gasto cardíaco contribuirían a la hipoperfusión coronaria con posterior daño miocárdico ${ }^{4}$.

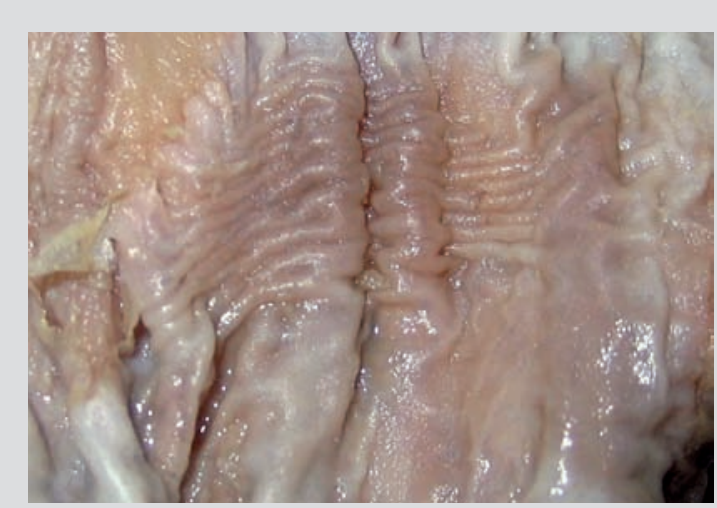

Figura 5.

Esófago anillado $(1 \mathrm{~cm})$ en el tercio distal.

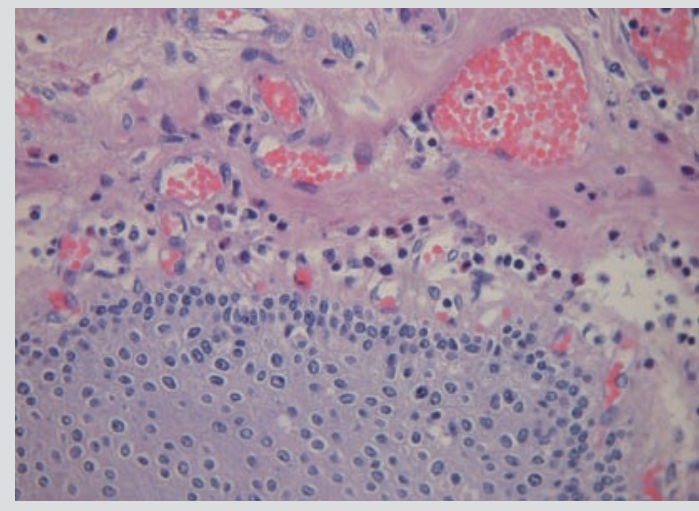

Figura 6.

Mucosa esofágica con acantosis, papilomatosis e infiltrado crónico con aislados eosinófilos intraepiteliales y más numerosos en lámina propia, HE 40x.
En nuestro caso, la presencia de un infiltrado inflamatorio con numerosos eosinófilos y mastocitos en el trombo, la placa subyacente, la pared muscular y la adventicia de la arteria coronaria debe hacernos sospechar la presencia de un componente alérgico de base y volver a realizar un estudio sistémico minucioso. En este caso, la patología asmática apoya esta sospecha, e incluso el esófago anillado o traquealizado que, aunque sin criterios histológicos, es típico de la esofagitis eosinófilica ${ }^{19}$ (asociada al asma). Puesto que el Síndrome de Kounis también ha sido descrito en episodios de asma ${ }^{20}$, parece probable, dado el antecedente de atopia, la clínica anterior al óbito y los hallazgos histológicos, que la erosión de la placa y posterior trombosis haya sido causada por un espasmo coronario secundario a una reacción alérgica.

Aunque los fenómenos alérgicos pueden causar muerte súbita, su diagnóstico se ve dificultado por la frecuente falta de datos clínicos en el momento de la realización de la autopsia y la imposibilidad de realizar un diagnóstico de certeza a posteriori, pues para demostrar un aumento mediadores en sangre es necesaria una recogida de muestras en condiciones especiales que no está implementada en el protocolo habitual. En nuestro caso, el antecedente de atopia 
no se conocía en el momento del estudio histopatológico, se confirmó en una entrevista familiar posterior y fue clave para orientar el cuadro hacia un síndrome de Kounis. Además del síndrome de Kounis, dentro de los mecanismos por los que los fenómenos alérgicos pueden desencadenar una muerte súbita destaca la anafilaxia, a veces desencadenada por reacción cruzada entre alérgenos, como los ácaros y proteínas de caracoles $^{21}$ (cita a), por ejemplo. Esta entidad debe ser sospechada en los casos de muerte súbita con corazón estructuralmente normal en individuos

\section{Bibliografía}

1. Kounis NG, Zavras GM. Histamine-induced coronary artery spasm: the concept of allergic angina. $\mathrm{Br} \mathrm{J}$ Clin Pract. 1991;45:121-8.

2. Nikolaidis LA, Kounis NG, Grandman AH. Allergic angina and allergic myocardial infarction: a new twist on an old syndrome. Can J Cardiol. 2002;18:508-11.

3. Biteker M. A new classification of Kounis syndrome. Int J Cardiol. 2010;145(3):553.

4. Ilhan E, Güvenç TS, Poyraz E et al. Kounis Syndrome secondary to cefuroxime axetil use in an asthmatic patient. Int J Cardiol. 2009; 137(3):e67-9.

5. Gázquez V, Dalmau G, Gaig P, et al. Kounis syndrome: report of 5 cases. J Investig Allergol Clin Immunol. 2010;20(2):162-5.

6. Cakar MA, Gündüz H, Kocayiğit I, et al. Acute coronary syndrome due to diclofenac potassium induced anaphylaxis: two Kounis syndrome variants in the same patient. Anadolu Kardiyol Derg. 2011;11(1):88.

7. Kounis NG, Giannopoulos S, Tsigkas GG. Eosinophilic responses to stent implantation and the risk of Kounis hypersensitivity associated coronary syndrome. Int J Cardiol. 2011 Jun 21 [Epub ahead of print].

8. Almpanis G, Siahos S, Karogiannis NC et al. Kounis syndrome: two extraordinary cases. Int J Cardiol $2011 ; 147(2):$ e35-8.

9. Wada $\mathrm{T}$, Abe $\mathrm{M}$, Yagi $\mathrm{N}$ et al. Coronary vasospasm secondary to allergic reaction following food ingestion: a case of type I variant Kounis syndrome. Heart Vessels. 2010;25(3):263-6.

10. Kogias JS, Sideris SK, Anifadis SK. Kounis syndrome associated with hypersensitivity to hymenoptera stings. Int J Cardiol. 2007 Jan 8;114(2):252-5.

11. Biteker M, Duran NE, Biteker FS et al. Allergic myocardial infarction in childhood: Kounis syndrome. Eur J Pediatr. 2010;169(1):27-9. con antecedentes alérgicos en los que, de otro modo, se recomendaría un estudio clínico familiar (e idealmente genético) para descartar la presencia de canalopatías.

Como dicen muchos expertos, el Síndrome de Kounis no es una enfermedad rara, pero sí es una enfermedad raramente diagnosticada. Debemos tenerla presente y descartarla ante una muerte súbita en el contexto de una reacción alérgica o de antecedentes de atopia, alergia o asma alérgico.

12. Kounis NG. Kounis syndrome (allergic angina and allergic myocardial infarction): A natural paradigm? Int J Cardiol 2006;110:7-14.

13. Fassio F, Almerigogna F. Kounis syndrome (allergic acute coronary syndrome): different views in allergologic and cardiologic literature. Intern Emerg Med. 2012 Jan 22 [Epub ahead of print].

14. Johnson JC, Jackson CL, Angelini GD, George S. Activation of matrix-degrating metalloproteinases by mast cell proteases in atherosclerotic plaques. Arterioscler Thromb Vasc Biol 1998;18:707-1715.

15. Laine P, Kaartinen M, Pentila A, Panula P, Paavonen T, Kovanen PT. Association between myocardial infarction and mast cells in the adventitia of the infarct-related coronary artery. Circulation 1999; 99:361-9.

16. Clejan S, Japa S, Clemetson C, Hasabnis SS, David $\mathrm{O}$, Talano JV. Blood histamine is associated with coronary artery disease, cardiac events and severity of inflammation and atherosclerosis. J Cell Mol Med 2002;6:583-92.

17. Takase B, Maruyama T, Kurita A, et al. Arachidonic acid metabolites in acute myocardial infarction. Angiology 1996;47:649-61.

18. Kaartinen M, Penttila A, Kovanen PT. Accumulation of activated mast cells in the shoulder region of human coronary atheroma, the prediction site of atheromatous rupture. Circulation 1994;90:1669-78.

19. Lucendo AJ, Carrión G, Navarro M et al. Eosinophilic esophagitis in adults, an emerging cause of dysphagia: Description of 9 cases. Rev Esp Enferm Dig. 2005; 97(4): 229-239.

20. Yanagawa Y, Nishi K, Tomiharu N. A case of takotsubo cardiomyopathy associated with Kounis syndrome. Int J Cardiol. 2009;132(2):e65-7.

21. Pajno GB, Morabito L, Barberio G. Allergy to house dust mite and snails: a model of cross-reaction between food and inhalant allergens with a clinical impact. Pediatr Pulmonol Suppl. 1999;18:163-4. 